\title{
Peroxisome proliferator-activated receptor gamma and its coactivator-1 alpha may be associated with features of the metabolic syndrome in adolescents
}

\author{
S Sookoian, S I García, P I Porto, G Dieuzeide', C D González² and C J Pirola \\ Cardiología Molecular, Instituto de Investigaciones Médicas A Lanari, Universidad de Buenos Aires, Buenos Aires, Argentina \\ ${ }^{1}$ CAIDEM, Chacabuco, Pcia de Buenos Aires, Argentina \\ ${ }^{2}$ Departamento de Farmacología, Facultad de Medicina, Universidad de Buenos Aires, Buenos Aires, Argentina
}

(Requests for offprints should be addressed to C J Pirola; Email: cjpirola@lanari.fmed.uba.ar)

\begin{abstract}
Our objective was to search for differences in genotypes of peroxisome proliferator-activated receptor gamma (PPAR $\gamma$ ) (Pro12 Ala) and its coactivator PGC-1 $\alpha$ (Gly482 Ser) in adolescents harboring features of metabolic syndrome. In a population-based study, we determined medical history, anthropometric variables, biochemical measurements and arterial blood pressures of 934 high-school students of Caucasian origin. We selected 220 adolescents who had systolic or diastolic blood pressures more than the 80th or less than the 20th percentiles based on the previous single set of measurements. One hundred and seventy-five adolescents completed the study and underwent two additional blood pressure measurements on different days, as well as biochemical analysis and genotyping. We found no association between insulin resistance, body mass index (BMI) and leptin levels and PPAR $\gamma$ and PGC-1 $\alpha$ genotypes. The 12 Ala PPAR $\gamma$ allele was associated with increased waist-to-hip ratio (WHR) and carriers seemed to have higher diastolic blood pressure and lower pulse pressure than non-carriers, particularly in the hypertensive and overweight group. Although Ser482 Ser PGC-1 $\alpha$ homozygotes had lower WHRs than other PGC-1 $\alpha$ genotypes, they were more frequent in the hypertensive group than in the normotensive (44.4 vs $24.5 \%, P<0.03)$, so the 482 Ser PGC-1 allele was in our population a risk factor for hypertension independently of WHR, homeostasis model assessment of insulin resistance, BMI and Pro12 Ala PPAR $\gamma$ variant (odds ratio=4.0, 95\% confidence interval 1.5-10.6, $P<0 \cdot 01$ ). Multiple regression analysis showed that age- and sex-adjusted systolic blood pressure correlated with the 482 Ser PGC-1 allele regardless of those covariates. In conclusion, the Gly482 Ser variant of the PGC-1 $\alpha$ gene may be an independent genetic risk factor for young-onset hypertension.
\end{abstract}

Journal of Molecular Endocrinology (2005) 35, 373-380

\section{Introduction}

Obesity in children and adolescents, defined as a body mass index (BMI) at or above the 95th percentile for children of the same age and sex (Himes \& Dietz 1994), has increased considerably. In this regard, it is known that approximately $60 \%$ of overweight adolescents have at least one additional risk factor for cardiovascular disease (Steinberger \& Daniels 2003). Furthermore, the metabolic syndrome, a clustering of combined manifestations of insulin resistance, hypertriglyceridemia, low high-density lipoprotein (HDL) cholesterol and hypertension, has emerged as a growing concern in the pediatric and adolescent population in recent years since this condition promotes the development of premature atherosclerosis (Goran \& Gower 1998, Weiss et al. 2004).

We have previously reported that in adolescents of European origin, hypertension was associated with an increased degree of central adiposity among other characteristics of the metabolic syndrome. Hypertensive students showed higher BMI, waist-to-hip ratio (WHR), triglycerides and insulin resistance and lower HDLcholesterol than normotensive ones. Furthermore, ageand sex-adjusted systolic arterial blood pressure (ABP) correlated with BMI, WHR insulin resistance and leptin (Porto et al. 2004).

Although significant progress has been made in elucidating the mechanisms underlying the metabolic syndrome, the genetic basis for the factors contributing to its clinical manifestations are largely unknown.

However, a number of candidate gene studies and genome scans have been performed, and genetic polymorphisms of the nuclear hormone receptor peroxisome proliferator-activated receptor gamma $(\operatorname{PPAR} \gamma)$ gene have been the focus of major interest. PPAR $\gamma$ is a transcription factor that belongs to the same family as steroid and thyroid hormone receptor (Desvergne \& Wahli 1999). It is activated by certain fatty acids, prostanoids and thiazolidinediones, a novel class of 
insulin-sensitizing antidiabetic agents (Olefsky 2000). Upon activation, PPAR $\gamma$ heterodimerizes with the retinoid $\mathrm{X}$ receptor and the heterodimer binds to specific PPAR-responsive elements of DNA to promote transcription of numerous target genes. The PPAR $\gamma$ gene is located on chromosome 3 and produces two different molecules, PPAR $\gamma 1$ and PPAR $\gamma 2$ by alternative mRNA splicing (Beamer et al. 1997). Although the isoform PPAR $\gamma 1$ is expressed in most tissues, PPAR $\gamma 2$ is specific for adipose tissue, where it plays a key role in regulating adipogenic differentiation (Auwerx 1999). A number of genetic variants have been identified, and one of the most studied polymorphism is the Prol2 Ala, which is the result of a CCA-to-GCA missense mutation in codon 12 of exon B of the PPAR $\gamma$ gene. This variant was associated with a decreased transcriptional activity in in vitro experiments (Yen et al. 1997). Furthermore, the Pro12 Ala genetic polymorphism has been associated with an increased risk of type 2 diabetes, obesity and insulin resistance.

In addition, PPAR $\gamma$ coactivator- 1 (PGC-1), a coactivator molecule recently identified based on its ability to interact with $\operatorname{PPAR} \gamma$, is involved in many important metabolic processes, including adaptive thermogenesis, mitochondrial biogenesis and fatty acid $\beta$-oxidation (Knutti \& Kralli 2001).

PGG-1 $\alpha$, a gene mapped to chromosome 4p15·1, exhibits a tissue-restricted expression pattern, and its expression is induced by physiological stimuli. For instance, it is expressed in high quantities in liver, heart, kidney and skeletal muscle, and also in adipose tissue, pancreas and brain (Larrouy et al. 1999).

Recently, a Gly482 Ser variant in exon 8 of the PCG-1 $\alpha$ gene was described (Ek et al. 2001) that is located in a part of the protein whose function is not known. Although the amino acid exchange at this position is conservative and does not create or eliminate protein motifs known to be functional, it was associated with type 2 diabetes in Danish, Japanese and Pima Indians (Ek et al. 2001, Hara et al. 2002, Muller et al. 2003).

We therefore decided to search for differences in genotypes of these two master genes associated with adipose tissue development and mitochondrial lipid oxidation, namely PPAR $\gamma 2$ (the Pro 12 Ala variant) and PGC-1 $\alpha$ (Gly482 Ser) in adolescents harboring features of the metabolic syndrome.

\section{Materials and methods}

\section{Population}

We have previously reported the prevalence of obesity and hypertension in a population-based study of 934 high-school students of Caucasian origin in an inner city of our country and we determined family history, anthropometric variables and $\mathrm{ABP}$ (Porto et al. 2004). Briefly, in a country town (Chacabuco, Province of Buenos Aires), we interviewed the total high-school student population consisting of 934 adolescents after written consent from their parents had been granted, in accordance with the procedures approved by the ethical committee of our institution. All the investigations performed in this study were conducted in accordance with the guidelines in The Declaration of Helsinki.

Under parental supervision, subjects responded to a questionnaire on medical history, medication and personal habits. Anthropometrical assessments included measurement of height, weight and waist and hip circumferences. Waist circumference was assessed in the standing position, midway between the highest point of the iliac crest and the lowest point of the costal margin in the mid-axillary line. Hip circumference was measured at the level of the femoral greater trochanter by the same observer. Body height and weight were recorded in light clothing, and BMI was computed as weight in kilograms divided by height in meters squared. Resting ABP was measured after subjects had been sitting for at least $30 \mathrm{~min}$. A mercury sphygmomanometer was used to measure blood pressure three times in the right arm by two investigators using cuffs with the appropriate length and width for the upper arm. We normalized BMI according to age and sex using the National Health and Nutrition Examination Survey located at the US Centers for Disease Control and Prevention web site (www.cdc.gov/nchs/about/major/ nhanes/growthcharts/datafiles.htm). Resting blood pressure was normalized as a Z-score according to sex and age using the US Task Force Tables (1996). After that, we selected and invited the participation of 220 adolescents who had systolic or diastolic blood pressures more than the 80 th or less than the 20th percentiles based on the previous single set of measurements. Totally 175 adolescents completed the study (105 were girls and 70 were boys) and underwent two additional blood pressure measurements on different days, and biochemical analysis and genotyping as described below. Adolescents with an average of systolic or diastolic blood pressure more than the 95th percentile and with no causes of secondary hypertension were considered essential hypertensives: 54 independent individuals met these criteria. These subjects were undiagnosed by the time this study was performed and therefore they did not receive any treatment for hypertension. Unfortunately, because there is no evidence-based definition that links specific levels of blood pressure with outcome, we used that 'statistical' definition of hypertension. Regarding overweight, we used age- and sex-adjusted BMI over the 85th percentile, which is considered a risk factor for obesity and hypertension in the adulthood (Dietz \& Bellizzi 1999). 


\section{Biochemical measurements}

Blood was drawn from fasting subjects who had been in a supine resting position for at least $30 \mathrm{~min}$. Plasma glucose, insulin, uric acid, total cholesterol, HDLcholesterol and low-density lipoprotein (LDL) cholesterol and triglycerides were measured by standard clinical laboratory techniques. We used the insulin-to-glucose ratio, the quantitative insulin sensitivity check index, and the homeostasis model assessment of insulin resistance (HOMA) (fasting insulin in microunits/ml multiplied by fasting glucose in $\mathrm{mmol} / \mathrm{l}$ divided by 22.5 ) as an estimation of insulin resistance. As HOMA appeared to show stronger correlations with the $Z$-score of systolic blood pressure $(r=0 \cdot 37, P<0 \cdot 05)$ we used the HOMA index as an indicator of insulin resistance. A commercial ELISA kit was used to measure plasma concentrations of leptin (Assay Designs, Inc., Ann Arbor, MI, USA) in blood samples collected with sodium EDTA.

\section{Genotyping}

The genetic analyses were done on genomic DNA extracted from white blood cells by a standard method as previously described (Kawasaki 1990).

Genotyping for PPAR $\gamma$ (Prol2 Ala) and PGG- $1 \alpha$ (Gly482 Ser) polymorphisms was performed by hot-start PCR-based restriction fragment length polymorphism analysis using molecular biology grade reagents unless indicated, and a Robocycler 96 thermal cycler (Stratagene, La Jolla, CA, USA).

Primers to detect the Gly482 Ser variant were 5'-CAA GTG CTG AGT CGT CAG-3', and 5'-GGG GTC TTT GAG AAA ATA AGG-3'; and genotyping employed digestion with MspI (New England BioLabs, Beverly, MA, USA) as described previously (Yen et al. 1997).

Primers to detect Prol2 Ala variant were 5'-GCG AAT TCA AGG CGA GTC-3', and 5'-GAT ATG TTT GCA GAC AGT GTA TCA GTG AAG GAA TGG CTT TGC G-3'; the variant was genotyped employing digestion with BstUI (New England BioLabs) as described elsewhere (Hara et al. 2002).

\section{Statistical analysis}

Quantitative data are expressed as means \pm S.E. Quantitative variable differences by disease state (normotensive vs hypertensive subjects) according to genotypes were studied by two-way ANOVA and individual mean differences were assayed by contrast vectors. Genotype frequencies were analyzed by means of a chi-square test.

Logistic and multiple regressions were used for testing of multivariate association between variables. We used the C.SS/Statistica program package from StatSoft (Tulsa, OK, USA) to perform these analyses. We considered significant a $P$ value $<0 \cdot 05$.

\section{Results}

In the whole sample, genotypes for PPAR $\gamma$ (Pro-Pro: 80.6\% and Pro-Ala: $19 \cdot 4 \%$ ) and PGC- $1 \alpha$ (Gly-Gly: $37 \cdot 4 \%$, Gly-Ser: $47 \cdot 1 \%$ and Ser-Ser: $15 \cdot 5 \%$ ) were in HardyWeinberg equilibrium and the frequencies observed are similar to those found in other Caucasian populations.

There was no difference in PPAR $\gamma$ Prol2 Ala genotypes between hypertensive (Pro-Pro: $79.6 \%$ and Pro-Ala: $20.4 \%$ ) and normotensive (Pro-Pro: $81.0 \%$ and Pro-Ala: $19 \cdot 0 \%$ ) adolescents. Hypertensive adolescents as a group showed many features of metabolic syndrome such as decreased HDL-cholesterol, elevated plasma levels of uric acid, triglycerides, fasting insulin and HOMA along with a higher WHR and age- and sexadjusted BMI (Table 1, see significance between groups).

In addition, we found no association between clinical characteristics of the adolescents according to PPAR $\gamma$ genotypes (Table 1), with two exceptions. The first was that the 12 Ala PPAR $\gamma$ allele was associated with a significantly increased WHR in normotensive subjects. Thus, the 12 Ala allele is a predictor of central obesity (odds ratio $(\mathrm{OR})=2 \cdot 37,95 \%$ confidence interval $(\mathrm{CI})$ $1 \cdot 05-5 \cdot 33, P<0 \cdot 03)$ independently of age, sex, hypertension, plasma leptin or insulin resistance. The second was that $12 \mathrm{Ala}$ allele carriers have a higher diastolic blood pressure and subsequently a lower pulse pressure than non-carriers, particularly in the hypertensive and overweight group (Fig. 1).

On the other hand, PGG-1 $\alpha 482$ Ser homozygotes had lower WHRs than the other PGG-1 $\alpha$ genotypes. There was an interaction between hypertension and the PGC-1 $\alpha$ genotype; homozygous PGC-1 $\alpha 482$ Ser hypertensive subjects had lower fasting insulin and HOMA than the other genotypes (Table 2). In fact, homozygous PGC-1 $\alpha 482$ Ser subjects were more frequent in the hypertensive group than in the normotensive one ( $44 \cdot 4$ vs $24.5 \%, P<0 \cdot 03)$, so the 482 Ser PGC- $1 \alpha$ allele was, in our population, a risk factor for hypertension independently of WHR, HOMA, age- and sex-adjusted BMI and Prol2 Ala PPAR $\gamma$ variant $(\mathrm{OR}=4 \cdot 0,95 \%$ CI $1 \cdot 5-10 \cdot 6, P<0 \cdot 01)$. In addition, multiple regression analysis showed that age- and sex-adjusted systolic blood pressure was correlated with the 482 Ser PGG-1 allele independently of those covariates (Table 3). The strong influence of BMI on systolic blood pressure explains why differences in systolic blood pressure between Gly482 allele carriers and non-carriers did not reach statistical significance in Table 2. In fact, in both, the overweight group (subjects with age- and sex-adjusted BMI over the 85th percentile) and the normal weight group, there were significant differences $(P<0 \cdot 03)$ in systolic blood pressure $Z$-score between carriers (overweight: 1.56 \pm $0 \cdot 22, n=44$, normal weight: $0 \cdot 01 \pm 0 \cdot 15, n=103)$ and non-carriers of the $P G C-1 a$ Gly482 (overweight: $2 \cdot 92 \pm 0 \cdot 0 \cdot 60, n=6$, normal weight: $0 \cdot 61 \pm 0 \cdot 32, n=21$ ). 
Table 1 Clinical and Biochemical characteristics of carriers and non-carriers of PPAR $12 \mathrm{Ala}$ in hypertensive and normotensive adolescents. Carriers of the PPAR $\gamma$ 12Ala correspond to Ala-Ala and Pro-Ala genotypes and non-carriers are subjects with Pro-Pro genotype. Results are expressed as means \pm S.E. Two-way ANOVA was performed for mean comparison between genotypes (non-carriers vs carriers) and groups (normotensives vs hypertensives) and these two-factor interactions. Particular mean differences were tested by contrast vectors

\begin{tabular}{|c|c|c|c|c|c|c|c|}
\hline & \multicolumn{2}{|c|}{ Normotensives } & \multicolumn{2}{|c|}{ Hypertensives } & \multicolumn{3}{|c|}{ Statistical significance ( $P$ value) } \\
\hline & Non-carriers & Carriers & Non-carriers & Carriers & Genotypes & Groups & Interaction \\
\hline$n$ & 98 & 24 & 37 & 10 & & & \\
\hline Age, years & $15 \cdot 72 \pm 0 \cdot 15$ & $15 \cdot 17 \pm 0 \cdot 30$ & $14 \cdot 62 \pm 0 \cdot 25$ & $15 \cdot 60 \pm 0 \cdot 47$ & NS & NS & NS \\
\hline Waist/hip Ratio & $0.79 \pm 0.01$ & $0.82 \pm 0.01^{*}$ & $0.83 \pm 0.01$ & $0.83 \pm 0.02$ & $0 \cdot 16$ & 0.0001 & NS \\
\hline Z-score BMI & $0 \cdot 10 \pm 0 \cdot 10$ & $0.34 \pm 0.21$ & $1 \cdot 00 \pm 0 \cdot 17$ & $1 \cdot 10 \pm 0.33$ & NS & 0.0001 & NS \\
\hline$Z$-score SABP & $-0.27 \pm 0.11$ & $0.02 \pm 0.22$ & $2 \cdot 62 \pm 0.18$ & $2 \cdot 39 \pm 0 \cdot 34$ & NS & 0.0001 & NS \\
\hline $\mathrm{HDL}(\mathrm{mmol} / \mathrm{l})$ & $1 \cdot 3 \pm 0 \cdot 1$ & $1 \cdot 3 \pm 0 \cdot 1$ & $1 \cdot 2 \pm 0 \cdot 1$ & $1 \cdot 2 \pm 0 \cdot 1$ & NS & 0.03 & NS \\
\hline LDL (mmol/L) & $2 \cdot 3 \pm 0 \cdot 1$ & $2 \cdot 5 \pm 0.1$ & $2 \cdot 5 \pm 0.1$ & $2 \cdot 5 \pm 0 \cdot 2$ & NS & NS & NS \\
\hline Triglycerides (mmol/l) & $0.96 \pm 0.06$ & $0.91 \pm 0.11$ & $1.19 \pm 0.09$ & $1 \cdot 19 \pm 0 \cdot 17$ & NS & 0.05 & NS \\
\hline Fasting glucose (mmol/l) & $4 \cdot 9 \pm 0 \cdot 1$ & $5 \cdot 0 \pm 0 \cdot 1$ & $5 \cdot 0 \pm 0 \cdot 1$ & $4 \cdot 8 \pm 0 \cdot 1$ & NS & NS & NS \\
\hline Fasting insulin (pmol/l) & $74 \pm 4$ & $76 \pm 9$ & $107 \pm 8$ & $103 \pm 14$ & NS & 0.001 & NS \\
\hline HOMA & $2 \cdot 31 \pm 0 \cdot 15$ & $2 \cdot 48 \pm 0.31$ & $3.52 \pm 0.25$ & $3.19 \pm 0.48$ & NS & 0.02 & NS \\
\hline
\end{tabular}

${ }^{*} P<0.05$ and ${ }^{* *} P<0.01$ with respect to non-carriers in the same group.

\section{Discussion}

The rapid increase in the prevalence of childhood overweight and its potential effect on cardiovascular morbidity and mortality emphasize the importance of identifying genetic traits that may help in the understanding of the factors contributing to the commonly occurring metabolic syndrome.

Therefore, in this study, we evaluated the role of genetic polymorphisms in two genes associated with adipose tissue development and mitochondrial lipid oxidation - PPAR $\gamma$ Pro12 Ala and PGG-1 $\alpha$ Gly482 Ser - in a population-based sample of adolescents.

Regarding central obesity and anthropometric variables, we found that PPAR 12 Ala variant was associated with an increase in WHR, particularly in the normotensive adolescents. To the best of our knowledge there is no report in the literature indicating such an effect in young populations. However, a similar increase in other indicators of central obesity in Ala allele bearers has been described in elderly subjects (Meirhaeghe $e t$ al. 2000).
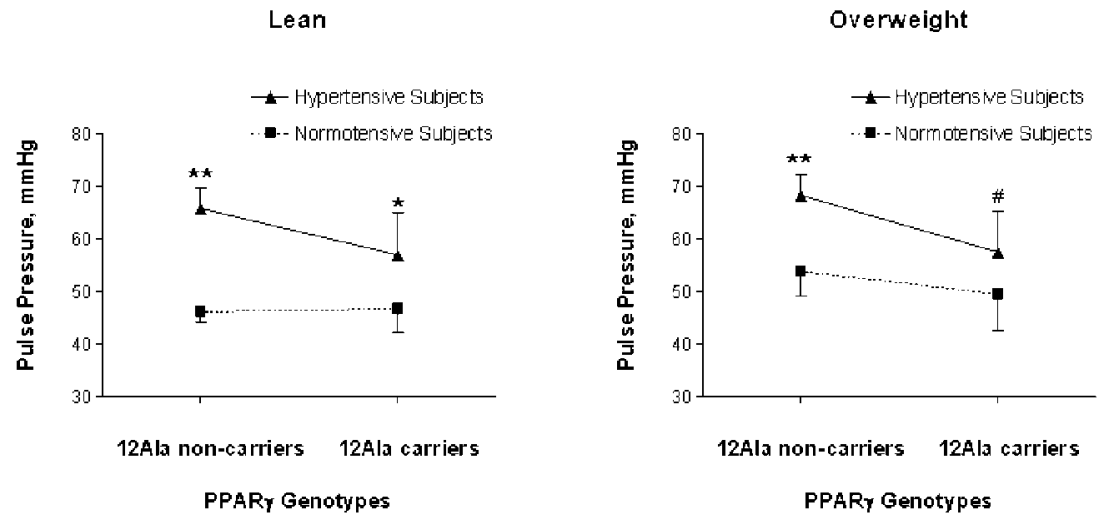

Figure 1 Pulse pressure in hypertensive (HT) and normotensive (NT) adolescent carriers and non-carriers of the PPAR 12 Ala variant, divided according to age- and sex-adjusted BMI as lean and overweight adolescents (carriers of the PPAR $12 \mathrm{Ala}$ correspond to Ala-Ala and Pro-Ala genotype subjects and non-carriers are subjects with Pro-Pro genotype). Results are expressed as means \pm S.D. ${ }^{*} P<0.05,{ }^{* *} P<0.001$ with respect to normotensive subjects with the same genotype. $\# P<0.03$ in comparison with hypertensive 12 Ala non-carriers. 
Table 2 Clinical and biochemical characteristics of carriers and non-carriers of PCG-1 Gly482 in hypertensive and normotensive adolescents. Carriers of the PGC-1 Gly482 correspond to Gly-Ser and Gly-Gly genotypes and non-carriers are subjects with Ser-Ser genotype. Results are expressed as means \pm S.E. Two-way ANOVA was performed for comparison between genotypes (carriers vs non-carriers of the PGC-1 Gly482) and groups (normotensives vs hypertensives) and the interaction between them. Particular mean differences were tested by contrast vectors

\begin{tabular}{|c|c|c|c|c|c|c|c|}
\hline & \multicolumn{2}{|c|}{ Normotensives } & \multicolumn{2}{|c|}{ Hypertensives } & \multicolumn{3}{|c|}{ Statistical significance ( $P$ value) } \\
\hline & Non-carriers & Carriers & Non-carriers & Carriers & Genotypes & Groups & Interaction \\
\hline$n$ & 14 & 108 & 12 & 34 & & & \\
\hline Age, years & $15 \cdot 2 \pm 0 \cdot 4$ & $15 \cdot 7 \pm 0 \cdot 1$ & $14 \cdot 8 \pm 0 \cdot 4$ & $14 \cdot 8 \pm 0 \cdot 4$ & NS & NS & NS \\
\hline Waist/hip Ratio & $0.77 \pm 0.01$ & $0.80 \pm 0.01$ & $0.81 \pm 0.02$ & $0.83 \pm 0.01$ & 0,002 & 0,04 & NS \\
\hline Z-score BMI & $-0.26 \pm 0.28$ & $0 \cdot 20 \pm 0 \cdot 1$ & $0.90 \pm 0.30$ & $1 \cdot 04 \pm 0 \cdot 18$ & NS & 0,0001 & NS \\
\hline$Z$-score SABP & $-0.29 \pm 0.29$ & $-0 \cdot 20 \pm 0 \cdot 10$ & $2 \cdot 85 \pm 0 \cdot 21$ & $2 \cdot 50 \pm 0 \cdot 19$ & NS & 0,0001 & NS \\
\hline Z-score DABP & $-0.39 \pm 0.20$ & $-0.38 \pm 0.07$ & $0.82 \pm 0.21$ & $0.66 \pm 0.13$ & NS & 0,0001 & NS \\
\hline Total cholesterol $(\mathrm{mmol} / \mathrm{l})$ & $4 \cdot 3 \pm 0 \cdot 2$ & $4 \cdot 0 \pm 0 \cdot 1$ & $4 \cdot 3 \pm 0 \cdot 2$ & $4 \cdot 2 \pm 0 \cdot 1$ & NS & NS & NS \\
\hline $\mathrm{HDL}(\mathrm{mmol} / \mathrm{l})$ & $1 \cdot 3 \pm 0 \cdot 1$ & $1 \cdot 3 \pm 0 \cdot 1$ & $1 \cdot 3 \pm 0 \cdot 1$ & $1 \cdot 2 \pm 0 \cdot 1$ & NS & 0,03 & NS \\
\hline $\mathrm{LDL}(\mathrm{mmol} / \mathrm{l})$ & $2 \cdot 4 \pm 0.2$ & $2 \cdot 3 \pm 0 \cdot 1$ & $2 \cdot 6 \pm 0 \cdot 2$ & $2 \cdot 5 \pm 0 \cdot 1$ & NS & NS & NS \\
\hline Triglycerides (mmol/l) & $1 \cdot 23 \pm 0 \cdot 15$ & $0.91 \pm 0.06$ & $0.98 \pm 0.16$ & $1 \cdot 26 \pm 0.09$ & NS & NS & 0,02 \\
\hline Fasting glucose $(\mathrm{mmol} / \mathrm{l})$ & $5 \cdot 1 \pm 0 \cdot 1$ & $4 \cdot 9 \pm 0 \cdot 1$ & $5 \cdot 0 \pm 0 \cdot 1$ & $5 \cdot 0 \pm 0 \cdot 1$ & NS & NS & NS \\
\hline Fasting insulin (pmol/l) & $79 \pm 12$ & $74 \pm 4$ & $80 \pm 13$ & $117 \pm 8^{*}$ & 0,11 & 0,02 & 0,02 \\
\hline HOMA & $2.55 \pm 0.4$ & $2 \cdot 31 \pm 0.14$ & $2 \cdot 64 \pm 0.43$ & $3 \cdot 75 \pm 0.26^{*}$ & 0,18 & 0,02 & 0,04 \\
\hline Uric acid $(\mu \mathrm{mol} / \mathrm{l})$ & $220 \pm 18$ & $232 \pm 6$ & $286 \pm 18$ & $278 \pm 12$ & NS & 0,0001 & NS \\
\hline Leptin ng/ml & $2 \cdot 88 \pm 1 \cdot 03$ & $2 \cdot 68 \pm 0 \cdot 37$ & $1 \cdot 82 \pm 1 \cdot 11$ & $4 \cdot 10 \pm 0 \cdot 66$ & NS & NS & NS \\
\hline
\end{tabular}

${ }^{*} P<0.05$ with respect to carriers in the same group.

In addition, in this report we found no association of the PPAR $\gamma$ genotypes with BMI. With reference to this finding, there are limited studies in adolescents. For instance, Witchel et al. (2001) addressed this issue in a small cohort of children with premature pubarche and adolescents with hyperandrogenism, reporting that there were no significant difference in BMI between Prol2 carriers and non-carriers. However, when subjects were followed up and BMI values were available at two time points, the rate of increase in BMI per year was significantly greater in the $12 \mathrm{Ala}$ carriers than noncarrier obese subjects, suggesting that 12 Ala allele may protect lean individuals from obesity while predisposing obese individuals to greater weight gain. In adults, a significant association with BMI was seen in some (Valve et al. 1999, Meirhaeghe et al. 2000, Koumanis et al. 2002) but not all studies (Evans et al. 2000, Kawasaki et al. 2002, Memisoglu et al. 2002). This difference may be explained by heterogeneous experimental design (for instance, case-control vs population-based studies), geographical origin of the subjects studies, and even nutritional influences (Luan et al. 2001). Although our study has a low power to discriminate BMI changes, consistent with the similar BMI between adolescents bearing the PPAR $\gamma$ Prol2 Ala variant, we observed no differences between plasma leptin levels in 12 Ala carriers in comparison with non-carriers, further indicating that the amount of leptin-secreting adipose tissue is not significantly different between both groups.

Second, regarding hypertension, we provide evidence that the PPAR 12 Ala variant was associated with a significant elevation of diastolic blood pressure in the whole sample. This finding, in the absence of any effect on systolic blood pressure, certainly implicates a significant drop in pulse pressure. In the last few years pulse pressure has emerged as an independent cardiovascular risk factor (Benetos 1999). Even though more experiments are necessary to confirm this association,

Table 3 Multiple regression summary for the dependent variable Z-score of systolic blood pressure in the sample of 169 adolescents

\begin{tabular}{|c|c|c|c|c|c|}
\hline & Beta & Beta S.E. & B & B S.E. & $P$ level \\
\hline Intercept & - & - & -1.469 & $1 \cdot 745$ & 0.4011 \\
\hline Waist/hip Ratio & 0.046 & 0.074 & 1.399 & $2 \cdot 244$ & 0.5340 \\
\hline HOMA & 0.184 & 0.073 & 0.192 & 0.076 & 0.0126 \\
\hline BMI Z-score & 0.438 & 0.072 & 0.652 & 0.107 & 0.0000 \\
\hline PPAR $\gamma$ 12Ala & 0.024 & 0.065 & 0.097 & 0.269 & 0.7181 \\
\hline PGC-1 Ser482Ser & 0.183 & 0.065 & 0.839 & 0.296 & 0.0052 \\
\hline PPAR $\gamma$ 12Ala $\times$ PGC-1 Ser482Ser & -0.003 & 0.069 & -0.043 & 1.053 & 0.967 \\
\hline
\end{tabular}


our results suggest that the 12 Ala variant may have a beneficial effect on the long-term cardiovascular risk in addition to its previously described decrease in the risk of type 2 Diabetes mellitus (Altshuler et al. 2000).

Third, with respect to insulin resistance, we found no effect of the 12 Ala variant on the HOMA index, indicating that the effect on this feature is modest if present at all at an early age. Again, although this result may be a consequence of low statistical power, a similar lack of association with indexes of insulin resistance was recently reported in young subjects (Poirier et al. 2000). Conversely, a prospective study, The Bogalusa Heart Study, reported that the 12 Ala variant attenuates the adverse association between adiposity and insulin resistance measures (Li et al. 2003).

One may speculate that these discrepancies may be related to the broad range of PPAR $\gamma$ actions ranging from an increase of PPAR $\gamma$ activity activating ligands that leads to increased insulin sensitivity (Olefsky 2000), to a reduction of receptor activity via germ line loss-of-function mutations, such as $\mathrm{P} 467 \mathrm{~L}$, leading to insulin resistance (Hegele 2003). In addition, the effect of altered activity of the PPAR $\gamma$ on whole-body insulin sensitivity has been appreciated for years. For instance, in both mice and humans, activating PPAR $\gamma$ ligands has beneficial effects on insulin sensitivity (Olefsky 2000).

As regards PGG-1 $\alpha$, it has been recognized as a important partner of PPAR $\gamma$ in transactivating several genes involved in mitochrondrial oxidative phosphorylation which are coordinately down-regulated in human diabetes (Mootha et al. 2003).

In our study, we found that adolescents carrying the Gly482 variant of the PGG- $1 \alpha$, without being diabetic, were more prone to have central obesity, elevated fasting insulin levels and HOMA index than the homozygous Ser-Ser. Although we found no reports in the literature about pediatric populations, our findings are in accord with what Muller et al. (2003) have found in non-diabetic Pima Indians, in whom they described that the Gly482 variant is associated with a decreased insulin secretory response to i.v. and oral glucose administration. Conversely, Ek et al. (2001) in Danish and Hara et al. (2002) in Japanese populations, found that overt type 2 diabetes is associated with the 482 Ser allele. Whether these discrepancies are due to different genetic population backgrounds, the time course of the disease (from modest insulin resistance to overt type 2 diabetes) or non-allelic interactions between different susceptibility and resistance genes remains to be further explored.

In addition, we found that Ser482 Ser $P G C-1 \alpha$ homozygotes were 2-fold more frequent in the hypertensive group than in the normotensive, indicating that the 482 Ser PGG-1 allele was, in our population, a predictor of hypertension independently of WHR, HOMA, BMI and Prol2 Ala PPAR $\gamma$ variant. Moreover, in the whole sample, age- and sex-adjusted systolic age blood pressure was also correlated with $P G C-1 \alpha 482$ Ser homozygosity regardless of others strong predictors of hypertension such as age and sex-adjusted BMI, WHR and insulin resistance. Surprisingly, even though PPAR $\gamma 2$ and PGC- $1 \alpha$ interact as transcription factors, we did not find any interaction between genotypes on systolic blood pressure Z-score.

By contrast, Andersen et al. (2005) and Oberkofler et al. (2003) studying mainly glucose-tolerant offspring of type 2 diabetes subjects or type 2 diabetes patients, recently reported a reduced risk of hypertension in $P G C-1 \alpha 482$ Ser carriers. The apparent discrepancy between the above mentioned findings and ours may be explained by the fact that the strong interaction between HOMA index and ABP is influenced by the PGC-1 $\alpha$ phenotypes, since we only observed a significant correlation between systolic ABP $Z$-score and HOMA in $P G C-1 \alpha$ Gly carriers $(P<0 \cdot 0001, r=0 \cdot 44)$. In other words, using HOMA as a covariate we confirmed that $P G C-1 \alpha 482$ Ser homozygotes showed a greater systolic ABP Z-score than $P G C$-1 $\alpha$ Gly carriers (data not shown).

From a physiological point of view, PGC- $1 \alpha$ seems to play a central role in both adipocyte differentiation and many important metabolic processes, including adaptive thermogenesis, mitochondrial biogenesis, and fatty acid $\beta$-oxidation (Knutti \& Kralli 2001). Although at first glance, it would seem that these variables are not connected with blood pressure regulation, it is worth mentioning that a mitochondrial gene mutation has been recently linked to a cluster of metabolic defects, including hypertension (Wilson et al. 2004).

Finally, these results taken together seem to indicate that PGC-1 $\alpha$ may have pleitropic effects beyond metabolic control. It can be argued, however, that other mechanisms, possibly involved in blood pressure elevation such as an increased sympathetic outflow, may help to decrease central obesity but increase insulin resistance in 482 Ser homozygous young subjects. However, this is the first evidence showing that in adolescents of European origin, a Gly482 Ser variant of the PGG- $1 \alpha$ gene may be an independent genetic risk factor for young-onset hypertension.

However, we cannot rule out the possibility that the association observed in our study is due to another functional polymorphism in linkage disequilibrium with the variant; thus the PGC- $1 \alpha$ may acts as a neutral molecular marker. Besides, further research is needed to confirm and extend the current findings, since the observations obtained from this study must face one of the main difficulties of association studies performed on complex traits - the small sample size.

In conclusion, we have found, for the first time as far as we know, that common variants in PPAR and $P G C-1 a$ are associated with features of the metabolic syndrome in Caucasian adolescents. Our experiments 
do not allow us to ascertain whether this is an epiphenomenon or if they are genetic risk factors for the early-onset of this common and complex disease. We hope that future experiments may solve this central question for public health.

\section{Acknowledgements}

This study was supported partially by grants B119 (Universidad de Buenos Aires) and PICT 05-08719 (Agencia Nacional de Promoción Científica y Tecnológica). S S, S I G and C J P belong to Consejo Nacional de Investigaciones Científicas y Técnicas. The authors declare that there is no conflict of interest that would prejudice the impartiality of this work.

\section{References}

Altshuler D, Hirschhorn JN, Klannemark M, Lindgren CM, Vohl MC, Nemesh J, Lane CR, Schaffner SF, Bolk S, Brewer C et al. 2000 The common PPARgamma Prol 2 Ala polymorphism is associated with decreased risk of type 2 diabetes. Nature Genetics $\mathbf{2 6}$ 76-80.

Andersen G, Wegner L, Jensen DP, Glumer C, Tarnow L, Drivsholm T, Poulsen P, Hansen SK, Nielsen EM, Ek J et al. 2005 PGG-1 alpha Gly482 Ser polymorphism associates with hypertension among Danish whites. Hypertension 45 565-570.

Auwerx J 1999 PPARgamma, the ultimate thrifty gene. Diabetologia 42 1033-1049.

Beamer BA, Negri C, Yen CJ, Gavrilova O, Rumberger JM, Durcan MJ, Yarnall DP, Hawkins AL, Griffin CA, Burns DK et al. 1997 Chromosomal localization and partial genomic structure of the human peroxisome proliferator activated receptor-gamma (hPPAR gamma) gene. Biochemical and Biophysical Research Communications 233 756-759.

Benetos A 1999 Pulse pressure and cardiovascular risk. Fournal of Hypertension 17 S21-S24.

Desvergne B \& Wahli W 1999 Peroxisome proliferator-activated receptors: nuclear control of metabolism. Endocrine Reviews $\mathbf{2 0}$ 649-688.

Dietz WH \& Bellizzi MC 1999 Introduction: the use of body mass index to assess obesity in children. American fournal of Clinical Nutrition 70 123S-125S.

Ek J, Andersen G, Urhammer SA, Gaede PH, Drivsholm T, Borch-Johnsen K, Hansen T \& Pedersen O 2001 Mutation analysis of peroxisome proliferator-activated receptor-gamma coactivator-1 (PGC-1) and relationships of identified amino acid polymorphisms to Type II diabetes mellitus. Diabetologia $\mathbf{4 4}$ 2220-2226

Evans D, Mann WA, Heer J, Michel U, Wendt D, Kortner B, Wolf A \& Beisiegel U 2000 Variation in the gene for human peroxisome proliferator activated receptor gamma (PPARgamma) does not play a major role in the development of morbid obesity. International Fournal of Obesity and Related Metabolic Disorders 24 647-651.

Goran MI \& Gower BA 1998 Abdominal obesity and cardiovascular risk in children. Coronary Artery Disease 9 483-487.

Hara K, Tobe K, Okada T, Kadowaki H, Akanuma Y, Ito C, Kimura S \& Kadowaki T 2002 A genetic variation in the PGG-1 gene could confer insulin resistance and susceptibility to Type II diabetes. Diabetologia 45 740-743.

Hegele RA 2003 Monogenic forms of insulin resistance: apertures that expose the common metabolic syndrome. Trends in Endocrinology and Metabolism 14 371-377.
Himes JH \& Dietz WH 1994 Guidelines for overweight in adolescent preventive services: recommendations from an expert committee. The Expert Committee on Clinical Guidelines for Overweight in Adolescent Preventive Services. American fournal of Clinical Nutrition 59 307-316.

Kawasaki ES 1990 Sample preparation from blood, cells, and other fluids. In PCR Protocols. A guide to Methods and Applications, pp 146-152. Eds MA Innis, DH Gelfand, JJ Sninsky \& TJ White. San Diego, CA: Academic Press, Inc.

Kawasaki I, Tahara H, Emoto M, Shoji T, Shioji A, Okuno Y, Inaba M \& Nishizawa Y 2002 Impact of Prol2 Ala variant in the peroxisome proliferator-activated receptor (PPAR) gamma 2 on obesity and insulin resistance in Japanese Type 2 diabetic and healthy subjects. Osaka City Medical Fournal 48 23-28.

Knutti D \& Kralli A 2001 PGG-1, a versatile coactivator. Trends in Endocrinology and Metabolism 12 360-365.

Koumanis DJ, Christou NV, Wang XL \& Gilfix BM 2002 Pilot study examining the frequency of several gene polymorphisms in a morbidly obese population. Obesity Surgery 12 759-764.

Larrouy D, Vidal H, Andreelli F, Laville M \& Langin D 1999 Cloning and mRNA tissue distribution of human PPARgamma coactivator-1. International fournal of Obesity and Related Metabolic Disorders 23 1327-1332.

Li S, Chen W, Srinivasan SR, Boerwinkle E \& Berenson GS 2003 The peroxisome proliferator-activated receptor-gamma2 gene polymorphism (Pro12 Ala) beneficially influences insulin resistance and its tracking from childhood to adulthood: the Bogalusa Heart Study. Diabetes 52 1265-1269.

Luan J, Browne PO, Harding AH, Halsall DJ, O'Rahilly S, Chatterjee VK \& Wareham NJ 2001 Evidence for gene-nutrient interaction at the PPARgamma locus. Diabetes 50 686-689.

Meirhaeghe A, Fajas L, Helbecque N, Cottel D, Auwerx J, Deeb SS \& Amouyel P 2000 Impact of the peroxisome proliferator activated receptor gamma2 Prol2 Ala polymorphism on adiposity, lipids and non-insulin-dependent diabetes mellitus. International Journal of Obesity and Related Metabolic Disorders 24 195-199.

Memisoglu A, Hankinson SE, Manson JE, Colditz GA \& Hunter DJ 2002 Lack of association of the codon 12 polymorphism of the peroxisome proliferator-activated receptor gamma gene with breast cancer and body mass. Pharmacogenetics 12 597-603.

Mootha VK, Lindgren CM, Eriksson KF, Subramanian A, Sihag S, Lehar J, Puigserver P, Carlsson E, Ridderstrale M, Laurila E et al. 2003 PGG-1 alpha-responsive genes involved in oxidative phosphorylation are coordinately downregulated in human diabetes. Nature Genetics 34 267-273.

Muller YL, Bogardus C, Pedersen O \& Baier L 2003 A Gly482 Ser missense mutation in the peroxisome proliferator-activated receptor gamma coactivator-1 is associated with altered lipid oxidation and early insulin secretion in Pima Indians. Diabetes $\mathbf{5 2}$ 895-898.

Oberkofler H, Holzl B, Esterbauer H, Xie M, Iglseder B, Krempler F, Paulweber B \& Patsch W 2003 Peroxisome proliferatoractivated receptor-gamma coactivator-1 gene locus: associations with hypertension in middle-aged men. Hypertension 41 368-372.

Olefsky JM 2000 Treatment of insulin resistance with peroxisome proliferator-activated receptor gamma agonists. Fournal of Clinical Investigation 106 467-472.

Poirier O, Nicaud V, Cambien F \& Tiret L 2000 The Prol2 Ala polymorphism in the peroxisome proliferator-activated receptor gamma2 gene is not associated with postprandial responses to glucose or fat tolerance tests in young healthy subjects: the European Atherosclerosis Research Study II. Fournal of Molecular Medicine 78 346-351.

Porto PI, Garcia SI, Dieuzeide G, Gonzalez C, Landa MS \& Pirola GJ 2004 Clinical features of the metabolic syndrome in adolescents: minor role of the Trp64 Arg beta3-adrenergic receptor gene variant. Pediatric Research 55 836-841. 
Steinberger J \& Daniels SR 2003 Obesity, insulin resistance, diabetes, and cardiovascular risk in children: an American Heart Association scientific statement from the Atherosclerosis, Hypertension, and Obesity in the Young Committee (Council on Cardiovascular Disease in the Young) and the Diabetes Committee (Council on Nutrition, Physical Activity, and Metabolism). Circulation 107 1448-1453.

US Task Force Tables 1996 Update on the 1987 Task Force Report on High Blood Pressure in Children and Adolescents: a working group report from the National High Blood Pressure Education Program. National High Blood Pressure Education Program Working Group on Hypertension Control in Children and Adolescents. Pediatrics 98 649-658.

Valve R, Sivenius K, Miettinen R, Pihlajamaki J, Rissanen A, Deeb SS, Auwerx J, Uusitupa M \& Laakso M 1999 Two polymorphisms in the peroxisome proliferator-activated receptor-gamma gene are associated with severe overweight among obese women. Fournal of Clinical Endocrinology and Metabolism 84 3708-3712.

Weiss R, Dziura J, Burgert TS, Tamborlane WV, Taksali SE, Yeckel CW, Allen K, Lopes M, Savoye M, Morrison J et al. 2004
Obesity and the metabolic syndrome in children and adolescents. New England Fournal of Medicine 350 2362-2374.

Wilson FH, Hariri A, Farhi A, Zhao H, Petersen KF, Toka HR, Nelson-Williams C, Raja KM, Kashgarian M, Shulman GI et al. 2004 A cluster of metabolic defects caused by mutation in a mitochondrial tRNA. Science 306 1190-1194.

Witchel SF, White C, Siegel ME \& Aston CE 2001 Inconsistent effects of the proline $12 \rightarrow$ alanine variant of the peroxisome proliferator-activated receptor-gamma 2 gene on body mass index in children and adolescent girls. Fertility and Sterility $\mathbf{7 6}$ $741-747$.

Yen CJ, Beamer BA, Negri C, Silver K, Brown KA, Yarnall DP, Burns DK, Roth J \& Shuldiner AR 1997 Molecular scanning of the human peroxisome proliferator activated receptor gamma (hPPAR gamma) gene in diabetic Caucasians: identification of a Prol2 Ala PPAR gamma 2 missense mutation. Biochemical and Biophysical Research Communications 241 270-274.

Received 25 May 2005

Accepted 17 June 2005 